\title{
Performance of a New Microfluidic Dengue NS1 Immuno-magnetic Agglutination Assay for the Rapid Diagnosis of Dengue Infection in Adults
}

\author{
Ekkarat Wongsawat, ${ }^{1}$ Yupin Suputtamongkol, ${ }^{1 *}$ Susan Assanasaen, ${ }^{1}$ Saowaluk Silpasakorn, ${ }^{1}$ Panisadee Avirutnan, ${ }^{2,3,4}$ \\ Chunya Puttikhunt, ${ }^{2,3,4}$ Nasikarn Angkasekwinai, ${ }^{1}$ Eakkawit Yamasmith, ${ }^{1}$ Tanapan Prommool, ${ }^{4}$ and Kanigar Niwattayakul ${ }^{5}$ \\ ${ }^{1}$ Division of Infectious Diseases and Tropical Medicine, Faculty of Medicine Siriraj Hospital, Mahidol University, Bangkok, Thailand; ${ }^{2}$ Division of \\ Dengue Hemorrhagic Fever Research, Faculty of Medicine Siriraj Hospital, Mahidol University, Bangkok, Thailand; ${ }^{3}$ Siriraj Center of Research \\ Excellence in Dengue \& Emerging Pathogens, Faculty of Medicine Siriraj Hospital, Mahidol University, Bangkok, Thailand; ${ }^{4}$ Molecular Biology of \\ Dengue and Flaviviruses Research Team, National Center for Genetic Engineering and Biotechnology, National Science and Technology \\ Development Agency, Klong Luang, Pathumthani, Thailand; ${ }^{5}$ Medical Department, Loei Provincial Hospital, Ministry of Health, Loei, Thailand
}

\begin{abstract}
Dengue (DENV) infections are a public health concern worldwide and thus early diagnosis is important to ensure appropriate clinical management. The rapid diagnostic test (RDT) targets nonstructural protein 1 (NS1) detection and is the main tool used for diagnostic purpose. In this study, we evaluated the performance of a new rapid and semiquantitative microfluidic DENV NS1 immuno-magnetic agglutination assay or IMA (ViroTrack Dengue Acute, BluSense Diagnostics, Copenhagen, Denmark). We studied 233 subjects confirmed to have DENV infection (by a real-time reverse transcriptase polymerase chain reaction) and 200 control samples were taken from patients with confirmed diagnoses of other febrile illnesses, in Thailand. Samples were tested using the NS1 antigen (Ag) detection methods: in-house NS1 Ag ELISA (ELISA), SD BIOLINE Dengue NS1 Ag RDT (ICT), and ViroTrack Dengue Acute (IMA). Sensitivities of these tests were $86.3 \%, 78.9 \%$, and $85.5 \%$, respectively. All tests showed high specificity $(100 \%$, 99\%, and $97 \%$ for ELISA, ICT, and IMA, respectively). The sensitivities of both RDTs were affected by the low sensitivity to DENV-2 and DENV-4. NS1 Ag was detected in every patient on day 1 and day 2 after onset of illness by ELISA and IMA with a decline in detection rates over time after day 6 of illness. NS1 detection rate using ICT decreased from $100 \%$ on day 1 of illness to $98.6 \%$ on day 2 after onset of illness. By day 6 , the detection rate was $45.9 \%$. Thus, IMA performed better than ICT for early and rapid diagnosis of DENV infections in endemic countries.
\end{abstract}

\section{INTRODUCTION}

Dengue, the most common arboviral infection, is caused by four distinct serotypes of the dengue virus (DENV-1, DENV-2, DENV-3, and DENV-4). It is endemic in over 100 countries with up to 100 million infections reported each year worldwide, resulting in 10,000 deaths. ${ }^{1,2}$ The symptoms of dengue infections vary from a mild and self-limited febrile illness to being potentially fatal due to severe hemorrhagic complications. ${ }^{2}$ No specific antiviral treatment is currently available. However, mortality from dengue can be reduced to almost zero by implementing timely and appropriate clinical management, which requires both early clinical and laboratory diagnosis. ${ }^{2}$

The nonstructural protein 1 (NS1) is a useful biomarker for early diagnosis. NS1 antigen (Ag) is secreted by DENVinfected cells and is detectable in blood from day 1 up to day 14 after onset of fever. ${ }^{3-5}$ Hence, many tests have been developed, which target the NS1 Ag, including the antigencapture enzyme-linked immune-absorbance assay (ELISA) and immunochromatographic test (ICT) or lateral flow antigen detection assays, also known as rapid diagnostic tests (RDTs). ${ }^{6}$ Although ELISA has displayed good analytical performance, it requires specialized laboratory equipment, trained staff, and takes hours to obtain results. The RDTs deliver results within minutes at lower costs, but they are usually less sensitive than ELISA and provide qualitative results. ${ }^{7-10}$ The ICT method for the rapid detection of NS1 Ag and/or DENV IgM, IgG is widely used in Thailand but data regarding its performance when compared with various DENV serotypes and duration of fever is scarce.

*Address correspondence to Yupin Suputtamongkol, Department of Medicine, Faculty of Medicine Siriraj Hospital, 2 Wang Lang, Bangkok- noi, Bangkok 10700, Thailand. E-mail: ysuputtamongkol@gmail.com
A rapid DENV NS1 immuno-magnetic agglutination assay (IMA) integrated with the microfluidic chip (ViroTrack Dengue Acute and BluBox, BluSense Diagnostics, Denmark) was recently developed and is commercially available. The NS1 detection method is based on the use of magnetic particles coated with a mix of monoclonal antibodies capable of detecting NS1 presented in all four DENV serotypes. A semiquantitative result, relative to the total amount of dengue NS1 Ag detected, is reported as positive, negative, or equivocal, in about 12 minutes. A recent study reported high analytical sensitivity and specificity of the assay, using serum samples from 135 travelers returning home after visiting dengue endemic countries. ${ }^{11}$ We reported herein the performance of this newly developed IMA test in Thailand, using samples from adult patients confirmed to be dengue positive by reverse-transcription polymerase chain reaction (RT-PCR).

\section{MATERIALS AND METHODS}

Samples of patients with dengue infection. A total of 581 EDTA plasma samples collected and archived previously were used in this study. The samples were taken from 233 adult patients enrolled in clinical studies related to dengue infection at Siriraj Hospital, in Bangkok and at Loei Hospital, in Loei Province, Thailand, between 2014 and $2017 .{ }^{12}$ These samples were collected daily during the admission process at both hospitals. The diagnosis of dengue was confirmed by RT-PCR in all patients. The dengue serotype was determined by both a reverse transcriptase quantitative PCR (RT-qPCR) protocol ${ }^{13}$ and NS1 serotype-specific ELISA (in-house NS1 Ag ELISA) test. ${ }^{14}$ Primary versus secondary infection were distinguished using DENV-specific IgG and IgM capture ELISA. ${ }^{15}$ The samples were stored in vial tubes at $-70^{\circ} \mathrm{C}$ until testing. Patients were informed about the use of their leftover samples for future research and The Ethical Committee at the Faculty Medicine 
Siriraj Hospital, Mahidol University, approved the study protocol (COA no: Si200/2019). This cohort study included a baseline sample (taken on admission date) and subsequent samples that were collected daily during hospitalization up to day 9 after onset of fever. The selection of the cohort was made considering a maximum of two consecutive days of negative results with the in-house NS1 Ag ELISA.

Samples were pre-characterized by RT-PCR, in-house NS1 $\mathrm{Ag}$, and SD BIOLINE Dengue NS1 Ag RDT (Bioline Diagnostics LLP, Delhi, India) when they were collected and were archived on unlinked anonymous basis. Demographic data and important clinical information such as duration of fever, sampling day, and serotypes were recorded.

Samples of patients with nondengue infection. We selected a set of 200 plasma samples collected on the admission date of adult patients who displayed acute febrile illness at Maharaj Nakhon Ratchasima Hospital, Loei Provincial Hospital, and Banmai Chaiyapod Hospital, in Buriram Province, and at Siriraj Hospital, in Bangkok, Thailand, between 2000 and 2018. These blood samples were collected as part of epidemiological and clinical studies of patients with acute undifferentiated fever. ${ }^{16,17}$ These studies included adult patients ( $>18$ years) who had acute fever (oral temperature $>38.0^{\circ} \mathrm{C}$ for less than 15 days) in the absence of an obvious focus of infection. In this study, we selected a set of samples from patients with confirmed diagnosis of other common causes of acute febrile illness that mimic dengue infection, including chikungunya, zika virus, influenza, leptospirosis, malaria, scrub typhus, murine typhus, and septicemia from various bacteria. Samples from patients with suspected co-infection were excluded. Laboratory tests to confirm for leptospirosis, scrub typhus, and murine typhus were carried out using the indirect immunofluorescent assay (IFA) as described previously. ${ }^{16,17}$ The Leptospira interrogans, serovar Autumnalis, pooled Orientia tsutsugamushi from Karp, Kato, and Gilliam strains, and Rickettsia typhi (Wilmington strain) were used as antigens for the detection of IgM and IgG antibodies for diagnosis of leptospirosis, scrub typhus, and murine typhus, respectively. The diagnosis of zika and chikungunya were confirmed by PCR. These samples had previously been precharacterized with both in-house NS1 Ag ELISA and SD BIOLINE Dengue NS1 Ag RDT. Thus, samples were tested for all of the listed pathogens.

Procedure. A total of 781 samples were tested with the ViroTrack Dengue Acute, following instructions provided by the manufacturer and the results were recorded including quantification of IMA units. In brief, after the initial self-check and calibration completed, step-by-step instructions were displayed on an LCD panel. Samples and patient data were entered into a system and saved. The IMA cartridge was removed from the cover and $30 \mu \mathrm{L}$ of plasma sample was loaded into the designated well. The cartridge was then inserted into the reader (BluBox; BluSense Diagnostics, Copenhagen, Denmark) where all the self-contained sample preparation, mixing, and reading steps took place. The display panel continued to show the progress of each run and after 8-10 minutes, it ended with a semi-quantitative report, namely, negative (green display, numerical result $<23$ ), equivocal (yellow display, range of numerical result 23.0-26.9 IMA units), positive (red display, range of numerical result 27.0-100.0 IMA units), and high positive (red display range of numerical result $>100.0$ IMA units). The machine had the capability to archive the reports. Samples with an "equivocal" result were re-tested, and these results were used in the performance analysis. Data from samples with an "equivocal result" after a re-test were discarded from the study (per the manufacturer's instruction). For SD BIOLINE Dengue NS1 Ag RDT, if the result displayed a "faint line," it was interpreted as a "positive" result in the study. Results were interpreted by two independent readers.

To exclude the likelihood of degradation of NS1 Ag over long-term storage of samples, samples that had an ELISA positive result and an IMA negative result were designated as "discordant" and re-tested with both ELISA and the ICT, and the most recent result was recorded.

Statistical analysis. Most dengue patients (85\%) in this study were admitted within 3 days of onset of fever and were hospitalized for supportive care, without any specific treatment until they became afebrile. Thus, each patient provided serial samples for the determination of sensitivity of NS1 detection for several days after onset of fever. We initially determined the overall sensitivities of the in-house NS1 Ag ELISA, ViroTrack Dengue Acute or IMA and SD BIOLINE Dengue NS1 Ag RDT or ICT for the diagnosis of DENV infection, using all the available samples. A two-by-two table was constructed to calculate the percentage of true-positive, false-positive, false-negative, and true-negative results. The standard diagnostic accuracy indices of the sensitivity and specificity with $95 \%$ confidence intervals (Cls) were calculated. Then subsequent analyses using in-house NS1 Ag ELISA as the gold standard were carried out to determine the sensitivities of ViroTrack Dengue Acute and SD BIOLINE Dengue NS1 Ag RDT as they were designed to detect NS1 Ag. The sensitivities of these tests were compared against various DENV serotypes and days since onset of fever. Cls for sensitivity and specificity were also calculated using the Exact Binomial method. ${ }^{18} \mathrm{McNemar} \chi{ }^{2}$ test was performed to determine the statistical difference in sensitivity and specificity between these tests and a $P$ value $<0.05$ (two-tailed) was considered to be statistically significant. ${ }^{19}$

\section{RESULTS}

In the dengue group, 581 samples from 233 patients ( $\mathrm{M}: \mathrm{F}=$ 132:101, median age was 24 and range 15-72 years) were tested. The median duration of illness prior to the hospital visit was 2 days (range 1-5 days). DENV-4 was the most commonly observed serotype during the collection period $(N=128$ or $54.9 \%$, all secondary infection), followed by DENV-3 ( $N=51$ or $21.9 \%$, with $11.5 \%$ primary infection), DENV-2 $(N=32$ or $12.7 \%$, with $8.8 \%$ primary infection), and DENV-1 ( $N=22$ or $9.4 \%$, with $13.6 \%$ primary infection).

Of the 200 patients in the control group, the diagnoses of patients included scrub typhus (60 patients, 30.0\%), murine typhus (41 patients, 20.5\%), leptospirosis (40 patients, $20 \%$ ), influenza infection (24 patients, $12 \%$ ), zika virus infection (18 patients, 9\%), bacteremia from various bacteria such as Escherichia coli (10 patients, 5\%), and malaria (7 patients, 3.5\%).

Assay performance. In total, nine equivocal results with the ViroTrack Dengue Acute were obtained and re-tested. The second results from the equivocal results were "positive" in two cases, and "negative" in four cases (false negative compared with in-house NS1 ELISA). The second "equivocal" 
result with ViroTrack Dengue Acute was obtained in three samples. As a result, 778 samples were included in the analysis of assay performance.

Based on SD BIOLINE Dengue NS1 Ag RDT, 19 samples (2, 11, 1, and 5 samples of DENV-1, DENV-2, DENV-3, and DENV-4, respectively) displayed a "faint line" and were interpreted as positive results. All samples, except one with the DENV-4 serotype, were positive by in-house NS1 ELISA. These 19 samples, except one with DENV-2 serotype, displayed a positive result with the ViroTrack Dengue Acute, with an IMA unit range from 37.58 to 76.3 .

Table 1 and Figure 1A show the comparisons of the overall sensitivity and the sensitivity of DENV-1, -2, -3, -4 NS1 Ag detection between in-house NS1 ELISA, ViroTrack Dengue Acute, and SD BIOLINE Dengue NS1 Ag RDT. In the dengue group, the overall sensitivity of the in-house NS1 ELISA was not statistically different from that of the ViroTrack Dengue Acute for diagnosis of DENV infection $(P=0.332)$. However, the overall sensitivity of the SD BIOLINE Dengue NS1 Ag RDT was significantly different from both in-house NS1 ELISA and ViroTrack Dengue Acute $(P<0.001$ and $P<0.001$, respectively). The overall sensitivity of ViroTrack Dengue Acute was lowest in patients with DENV-2, while the sensitivities of SD BIOLINE Dengue NS1 Ag RDT were less than $80 \%$ in DENV-2 and DENV-4. The specificity was $100 \%(95 \% \mathrm{Cl}$ 98.2-100\%) for in-house ELISA NS1 test, 99\% $(95 \% \mathrm{Cl}$ 96.4-99.9\%) for SD BIOLINE Dengue NS1 Ag RDT, and 97\% (95\% Cl 93.6-98.9\%) for ViroTrack Dengue Acute. False positives by the ViroTrack Dengue Acute were seen in three patients with scrub typhus, two patients with murine typhus, and one patient with influenza. False positives of SD BIOLINE Dengue NS1 Ag RDT occurred in two samples with scrub typhus.

When we compared the sensitivity of ViroTrack Dengue Acute and SD BIOLINE Dengue NS1 Ag RDT with in-house ELISA NS1 assay, ViroTrack Dengue Acute and SD BIOLINE Dengue NS1Ag RDT displayed an overall sensitivity of $97.8 \%(95 \% \mathrm{Cl} 96.1-98.9 \%)$ and $91.2 \% \quad(95 \% \mathrm{Cl}$ 88.3-93.5\%), respectively (Figure 1B).

Days-of-fever sensitivity analysis. Table 2 shows the distribution of cumulative samples taken over various days after onset of illness, various DENV serotypes, and sensitivities of NS1 Ag detection by in-house ELISA. NS1 Ag was detected in all samples at day 1 and day 2 after fever onset, after which we observed a decline in detection rate. However, NS1 Ag remained detectable in two-thirds of samples between day 7 and day 9 . Figure $2 A$ shows the comparison of the sensitivities of in-house ELISA NS1 Ag test, ViroTrack Dengue Acute, and SD BIOLINE NS1 Ag RDT several days after fever onset. Meanwhile Figure 2B shows a comparison of the sensitivities of ViroTrack Dengue Acute or SD BIOLINE NS1 Ag RDT with in-house ELISA NS1 Ag test over the days since onset of fever. The sensitivity of the SD BIOLINE NS1 Ag RDT was significantly lower than that of the ViroTrack Dengue Acute after day $5(45.9 \%, 95 \% \mathrm{Cl} 36.3-55.7 \%$ and $64.2 \%, 95 \% \mathrm{Cl}$ $54.5-73.2 \%$, respectively, $P=0.0011$ ).

\section{DISCUSSION}

The detection of DENV RNA by RT- PCR provides definitive diagnosis of DENV infection; however, DENV viremia quickly become undetectable after onset of fever. ${ }^{7}$ As a result, detection of the NS1 antigen, either by ELISA or by ICT, is widely used to diagnose DENV infection. However, the sensitivity of the NS1 tests varies according to the study population and study design, with the sensitivities ranging from $60 \%$ to $95 \%$ for ELISA and from $40 \%$ to $80 \%$ for ICT. $^{3-6}$ The sensitivity was consistently lower in secondary infection than primary infection and in DENV-2 serotype compared with the other serotypes. Overall, this study was conducted in adults, most of whom had secondary dengue infection with DENV-4 and DENV-3 as the most common serotypes. The distribution of infective serotypes correlated to the distribution of DENV serotypes in Thailand during the study period. In this study, the specificity of NS1 detection assays was tested in samples, collected before and during the period we collected samples from patients with dengue because the incidence of other febrile illnesses, such as leptospirosis, scrub typhus, was less than DENV infection. ${ }^{14,15}$ We confirmed that NS1 detection by the ELISA method demonstrated excellent sensitivity and specificity during the early phase of infection for all four DENV serotypes.

TABLE 1

Overall results and sensitivity for each test, using DENV RT-PCR as the gold standard for final diagnosis

\begin{tabular}{|c|c|c|c|c|c|c|}
\hline & \multicolumn{5}{|c|}{ DENV infection } & \multirow[b]{2}{*}{ Other diseases } \\
\hline & Total & DENV-1 & DENV-2 & DENV-3 & DENV-4 & \\
\hline $\begin{array}{l}\text { Number of samples tested } \\
\text { In-house ELISA }\end{array}$ & 578 & 50 & 77 & 115 & 336 & 200 \\
\hline Positive & 499 & 45 & 68 & 103 & 283 & 0 \\
\hline Negative & 78 & 5 & 9 & 12 & 53 & 200 \\
\hline Sensitivity, \% & 86.3 & 90.0 & 88.3 & 89.6 & 84.2 & $\mathrm{n} / \mathrm{a}$ \\
\hline $95 \% \mathrm{Cl}$ & $83.3-89.0$ & $78.2-96.7$ & 78.9-94.5 & $82.5-94.5$ & 79.9-88.0 & $\mathrm{n} / \mathrm{a}$ \\
\hline \multicolumn{7}{|l|}{ ViroTrack Dengue Acute } \\
\hline Positive & 494 & 45 & 61 & 104 & 284 & 6 \\
\hline Negative & 84 & 5 & 16 & 11 & 52 & 194 \\
\hline Sensitivity, \% & 85.5 & 90.0 & 79.2 & 90.4 & 84.5 & $\mathrm{n} / \mathrm{a}$ \\
\hline $95 \% \mathrm{Cl}$ & 79.9-88.0 & $78.2-96.7$ & $68.5-87.6$ & $83.5-95.1$ & $80.2-88.2$ & $\mathrm{n} / \mathrm{a}$ \\
\hline \multicolumn{7}{|l|}{ SD BIOLINE NS1 Ag RDT } \\
\hline Positive & 456 & 43 & 58 & 97 & 258 & 2 \\
\hline Negative & 122 & 7 & 19 & 18 & 78 & 188 \\
\hline Sensitivity, \% & 78.9 & 86.0 & 75.3 & 84.3 & 76.8 & $\mathrm{n} / \mathrm{a}$ \\
\hline $95 \% \mathrm{Cl}$ & $75.3-82.2$ & $73.3-94.2$ & $64.2-84.4$ & $76.4-90.5$ & $71.9-81.2$ & $\mathrm{n} / \mathrm{a}$ \\
\hline
\end{tabular}


A

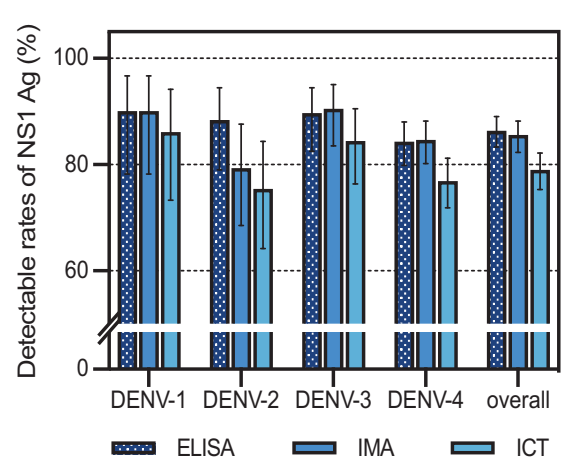

B

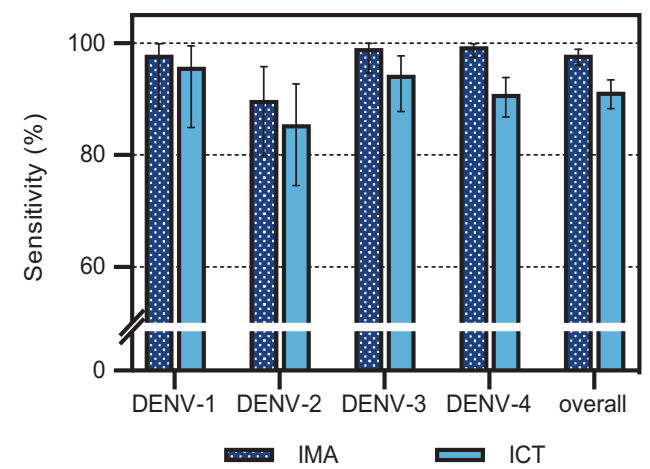

FIGURE 1. (A) Overall detectable rates of NS1 Ag by in-house ELISA (ELISA), ViroTrack Dengue Acute (IMA), and SD BIOLINEDengue NS1 RDT (ICT), for different DENV serotypes (DENV-1 to DENV- 4). The error bars represent the upper and lower limit of the $95 \%$ Cl. (B) Detectable rates of NS1 Ag (sensitivities) by IMA and ICT for different DENV serotypes compared with in- house ELISA. The error bars represent the upper and lower limit of the $95 \% \mathrm{Cl}$. This figure appears in color at www.ajtmh.org.

The ViroTrack Dengue Acute or IMA assay was recently developed for the detection of NS1 antigens. Recently published studies show that the sensitivity and specificity of IMA are comparable to that of ELISA and ICT in dengue patients who live in endemic areas ${ }^{10,20}$ and/or travelers returning home after a visiting endemic area. ${ }^{11}$ The results of our study confirmed the high sensitivity of this novel semi-quantitative technology among adult patients with dengue in Thailand. The sensitivity of IMA was comparable to that of ELISA and superior than the ICT. The positive predictive value (PPV) of all three NS1 Ag detection assays tested here is likely to be $99 \%$ or higher, given the high specificities observed (ranging from $97 \%$ to $100 \%$ ). We determined no cross reaction with zika infection in 18 samples in this study, which was consistent with previous publications that stated that zika virus NS1 was not likely to present a cross-reactivity problem in DENV NS1 assays. ${ }^{21}$ We did not evaluate the cross-reactivity of these tests with samples from patients with chikungunya. However, results from a previous study demonstrated that there was no cross reaction of various NS1 assays, including the ICT used in this study. ${ }^{22}$ Therefore, individuals testing positive in an endemic country are unlikely to require further confirmatory testing.

However, after day 5 of fever onset, the rate of NS1 detection decreased to only two-thirds in both ELISA and IMA and by half in ICT. Consequently, individuals who tested negative on either ELISA or RDTs but presented clinical symptoms of dengue should be re-tested, or their diagnosis should be confirmed by other laboratory tests such as a RT-PCR or $\operatorname{lgM}$ and IgG detection. Data from other studies evaluating NS1 tests show that adding the RT-PCR diagnostic test after day 5 does not significantly increase the number of positive detected; however, additional antibody testing does. Moreover, detection of positive dengue cases increases with the incorporation of IgM testing starting on day 4 after fever onset when NS1 detection begins to decrease. ${ }^{20}$

Another limitation of both the IMA and ICT method in comparison to ELISA is its lower sensitivity to the DENV-2 serotype. Previous reports have shown low detection rates for DENV-4 and DENV-2, 30\% and 60\%, respectively, using RDTs or ELISA tests. ${ }^{23,24}$ We saw evidence of a slightly lower sensitivity for DENV-2 using IMA and for DENV-2 and DENV-4 using ICT in this study. However, they were fewer samples of DENV-1 and DENV-2 than DENV-3 and DENV-4. Thus, further studies are necessary to evaluate the performance of this test among DENV-1 and DENV-2 serotypes.

In conclusion, our results confirmed that the IMA that provides automatic reading and quantification is effective for early diagnosis of dengue infection in adult patients in endemic regions including Thailand. The assay is rapid and has similar performance capabilities to the ELISA method. The high level of agreement between the results tested on capillary, venous, and serum samples was also recently revealed in a study from Malaysia. ${ }^{10}$ Therefore, the application of this rapid test can lead to better management of DENV infections in resourcelimited or endemic areas where a number of symptomatic

TABLE 2

The NS1 Ag detection rates, using the in-house ELISA NS1 Ag test for four DENV serotypes, up to 9 days after onset of fever

\begin{tabular}{|c|c|c|c|c|c|c|}
\hline \multirow{2}{*}{$\begin{array}{l}\text { Days since } \\
\text { onset of fever }\end{array}$} & \multirow[b]{2}{*}{ Total, $n(\%)$} & \multicolumn{5}{|c|}{ Positive NS1 Ag, $n$ (\%) } \\
\hline & & Total & DENV-1 & DENV-2 & DENV-3 & DENV-4 \\
\hline Day 1 & $29(5)$ & $29(100)$ & $1(100)$ & $2(100)$ & 8 (100) & $18(100)$ \\
\hline Day 2 & $112(19.4)$ & $112(100)$ & $9(100)$ & $13(100)$ & $28(100)$ & $62(100)$ \\
\hline Day 3 & $131(22.7)$ & $128(97.7)$ & $15(93.8)$ & $16(100)$ & $26(100)$ & 71 (91.3) \\
\hline Day 4 & $107(18.5)$ & 93 (86.9) & $8(88.9)$ & $13(86.7)$ & $16(94.1)$ & $56(84.8)$ \\
\hline Day 5 & $90(15.6)$ & $64(71.7)$ & $7(87.5)$ & $9(64.3)$ & $10(66.7)$ & $38(71.7)$ \\
\hline Day 6 & 65 (11.2) & $44(67.7)$ & $5(83.3)$ & $8(80)$ & 7 (58.3) & $24(64.9)$ \\
\hline Days $7-9$ & $44(7.6)$ & 29 (65.9) & $0(0)$ & 7 (100) & 8 (88.9) & 14 (51.9) \\
\hline Total & 578 (100) & 499 (86.3) & $45(90)$ & 68 (88.3) & 103 (89.6) & 283 (84.2) \\
\hline
\end{tabular}


A

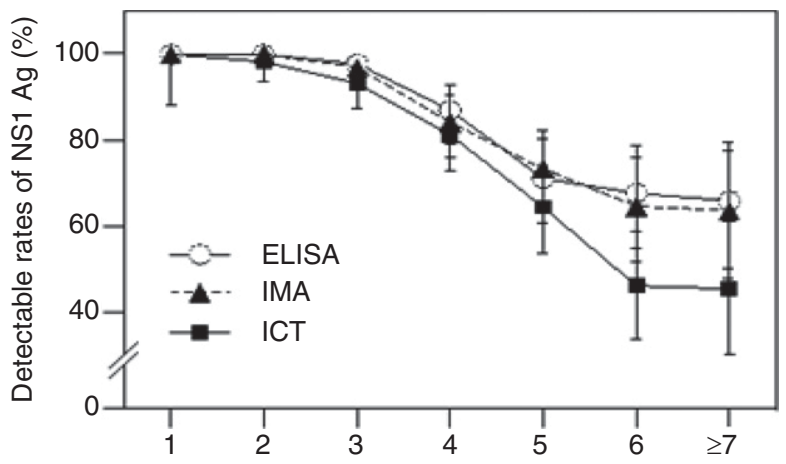

Days after onset of fever
B

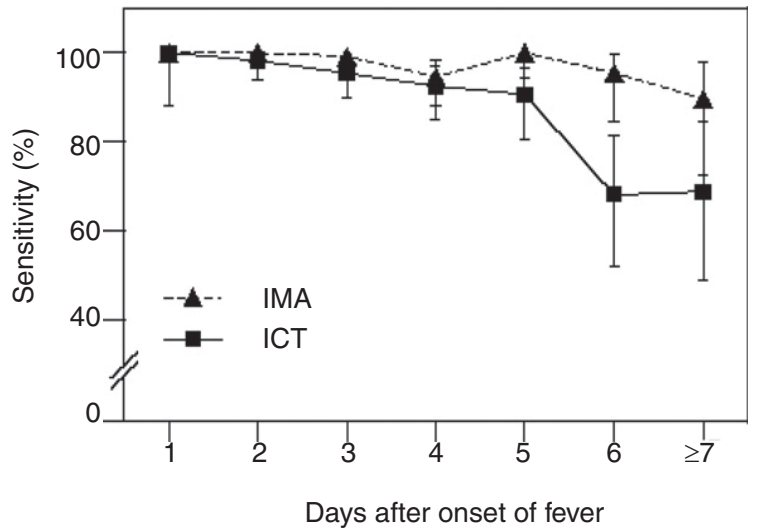

FIGURE 2. (A) Overall detectable rates of NS1 Ag by ELISA, IMA, and ICT over a range of days after onset of fever. The error bars represent the upper and lower limits of the $95 \%$ Cl. (B) Detectable rates (sensitivities) of NS1 Ag by IMA, and ICT over a range of days after onset of fever compared with in-house ELISA. The error bars represent the upper and lower limits of the $95 \% \mathrm{Cl}$.

patients can progress to DHF and/or the much worse DSS. Thus, there is an urgent need to determine and develop point of care detection of biomarker that correlates with DENV severity.

Received December 8, 2020. Accepted for publication May 11, 2021. Published online July 19, 2021.

Acknowledgments: We acknowledge the help and support provided by Drs. Filippo Bosco and Laura C. Bohorquez. BluSense Diagnostics ApS, Denmark, provided the ViroTrack Dengue Acute and funding support to acquire ICT used in this study. The funders also provided advice for study design, statistical analysis, and preparation of the manuscript.

Disclosure: The Ethical Committee at the Faculty Medicine Siriraj Hospital, Mahidol University, approved the study protocol (COA no: Si200/ 2019).

Authors' addresses: Ekkarat Wongsawat, Yupin Suputtamongkol, Susan Assanasaen, Saowaluk Silpasakorn, Nasikarn Angkasekwinai, and Eakkawit Yamasmith, Department of Medicine, Faculty of Medicine Siriraj Hospital, Mahidol University, Thailand, E-mails: ewongsawat@gmail.com, ysuputtamongkol@gmail.com, susan. ass@mahidol.ac.th, saowaluksilpasakorn@gmail.com, nasikarn@ gmail.com, and mike_th99@hotmail.com. Panisadee Avirutnan, Siriraj Center of Research Excellence in Dengue \& Emerging Pathogens, Faculty of Medicine Siriraj Hospital, Mahidol University, Thailand, E-mail: panisadee.avi@mahidol.edu. Chunya Puttikhunt, Molecular Biology of Dengue and Flaviviruses Research Team, National Center for Genetic Engineering and Biotechnology, National Science and Technology Development Agency, Klong Luang, Pathumthani, Thailand, E-mail: chaunyapk@biotec.or.th.Tanapan Prommool, Molecular Biology of Dengue and Flaviviruses Research Team, National Center for Genetic Engineering and Biotechnology, National Science and Technology Development Agency, Klong Luang, Pathumthani, Thailand, E-mail: tanapan.pro@biotec.or.th. Kanigar Niwattayakul, Loei Hospital, Loei Province, Thailand, E-mail: janthira1817@gmail.com.

\section{REFERENCES}

1. Shepard DS, Undurraga EA, Halasa YA, Stanaway JD, 2016. The global economic burden of dengue: a systematic analysis. Lancet Infect Dis 16: 935-941. doi: 10.1016/S1473-3099(16) 00146-8.

2. World Health Organization, 2012. Global strategy for dengue prevention and control, 2012-2020. Available at: https://www.who. int/ denguecontrol/9789241504034/en/. Accessed October 2019.

3. Alcon S, Talarmin A, Debruyne M, Falconar A, Deubel V, Flamand $M, 2002$. Enzyme-linked immunosorbent assay specific to dengue virus type 1 nonstructural protein NS1 reveals circulation of the antigen in the blood during the acute phase of disease in patients experiencing primary or secondary infections. J Clin Microbiol 40: 376-381.

4. Guzman MG et al., 2010. Dengue: a continuing global threat. Nat Rev Microbiol 8 (Suppl): S7-S16. doi: 10.1038/nrmicro2460.

5. Hu D, Di B, Ding X, Wang Y, Chen Y, Pan Y, Wen K, Wang M, 2011. Kinetics of non-structural protein 1, IgM and IgG antibodies in dengue type 1 primary infection. Virol J 47. doi: 10.1186/ 1743-422X-8-47.

6. Casenghi M, Kosack C, Li R, Bastard M, Ford N, 2018. NS1 antigen detecting assays for diagnosing acute dengue infection in people living in or returning from endemic countries. Cochrane Database Syst Rev 5: 2-5. doi: 10.1002/14651858.CD011155. pub2.

7. Hang VT et al., 2009. Diagnostic accuracy of NS1 ELISA and lateral flow rapid tests for dengue sensitivity, specificity and relationship to viraemia and antibody responses. PLoS Negl Trop Dis 3: e360. doi: 10.1371/journal.pntd.0000360.

8. Pal S, Dauner AL, Mitra I, Forshey BM, Garcia P, Morrison AC, Halsey ES, Kochel TJ, Wu S-JL, 2014. Evaluation of dengue NS1 antigen rapid tests and ELISA kits using clinical samples. PLoS One 9: e113411. doi: 10.1371/journal.pone.0113411.

9. Raafat N, Blacksell SD, Maude RJ, 2019. A review of dengue diagnostics and implications for surveillance and control. Trans $R$ Soc Trop Med Hyg 113: 653-660. doi: 10.1093/trstmh/trz068.

10. Chong ZL, Sekaran SD, Soe HJ, Peramalah D, Rampal S, Ng CW, 2020. Diagnostic accuracy and utility of three dengue diagnostic tests for the diagnosis of acute dengue infection in Malaysia. BMC Infect Dis 20: 210. doi: 10.1186/s12879-0204911-5.

11. Alejo-Cancho I, Navero-Castillejos J, Peiró-Mestres A, Albarracín R, Barrachina J, Navarro A, Gonzalo V, Pastor V, Muñoz J, Miguel JM, 2020. Evaluation of a novel microfluidic immunomagnetic agglutination assay method for detection of dengue virus NS1 antigen. PLoS Negl Trop Dis 14: e0008082. doi: 10. 1371/journal.pntd.0008082.

12. Suputtamongkol $Y$ et al., 2021. Ivermectin accelerates circulating nonstructural protein 1 (NS1) clearance in adult dengue patients: a combined phase 2/3 randomized double-blinded placebo controlled trial. Clin Infect Dis 72: e586-e593. doi: 10. 1093/cid/ciaa1332.

13. Shu PY, Chang SF, Kuo YC, Yueh YY, Chien LJ, Sue CL, Lin TH, Huang JH, 2003. Development of group- and serotype-specific one-step SYBR green 1-based real-time reverse transcriptionPeR assay for dengue virus. J Clin Microbiol 41: 2408-2416. 
14. Puttikhunt $C$ et al., 2011. The development of a novel serotypingNS1-ELISA to identify serotypes of dengue virus. J Clin Virol 50: 314-319. doi: 10.1016/j.jcv.2011.01.001.

15. Innis BL, Nisalak A, Nimmannitya S, Kusalerdchariya S, Chongswasdi V, Suntayakorn S, Puttisri P, Hoke CH, 1989. An enzyme-linked immunosorbent assay to characterize dengue infections where dengue and Japanese encephalitis co-circulate. Am J Trop Med Hyg 40: 418-427.

16. Suttinont $C$ et al., 2006. Causes of acute undifferentiated febrile illness in rural Thailand: a prospective observational study. Ann Trop Med Hyg 100: 363-370.

17. Thipmontree W, Suputtamongkol $Y$, Tantibhedhyangkul W, Suttinont C, Wongsawat E, Silpasakorn S, 2014. Human leptospirosis trends: northeast Thailand, 2001-2012. Int J Environ Res Public Health 11: 8542-8551. doi: 10.3390/ijerph110808542.

18. Clopper CJ, Pearson ES, 1934. The use of confidence or fiducial limits illustrated in the case of the binomial. Biometrika 26: 404413.

19. Trajman A, Lui ZRR, 2008. McNemar $\chi^{2}$ test revisited: comparing sensitivity and specificity of diagnostic examinations. Scand $J$ Clin Lab Invest 68: 77-80.
20. Roy P, Gaur R, Anand A, 2019. Evaluation of a novel immunomagnetic assay technology for rapid detection of dengue NS1 antigen. Inter J Res Med Sci. 8: 129. doi: 10.18203/23206012.jirms20195895.

21. Matheus S, Boukhari R, Labeau B, Ernault V, Bremand L, Kazanji $M$, Rousset D, 2016. Specificity of dengue NS1 antigen in differential diagnosis of dengue and Zika virus infection. Emerg Infect Dis 22: 1691-1693. doi: 10.3201/eid2209.160725.

22. Lee $\mathrm{H}$ et al., 2019. Comparison of six commercial diagnostic tests for the detection of dengue virus non-structural-1 antigen and IgM/IgG antibodies. Ann Lab Med 39: 566-571. doi: 10.3343/ alm.2019.39.6.566.

23. Bessoff K, Delorey M, Sun W, Hunsperger E, 2008. Comparison of two commercially available dengue virus (DENV) NS1 capture enzyme-linked immunosorbent assays using a single clinical sample for diagnosis of acute DENV infection. Clin Vaccine Immunol 15: 1513-1518. doi: 10.1128/CVI.00140-08.

24. Ramirez AH, Moros Z, Comach G, Zambrano J, Bravo L, Pinto B, Vielma S, Cardier J, Liprandi F, 2009. Evaluation of dengue NS1 antigen detection tests with acute sera from patients infected with dengue virus in Venezuela. Diagn Microbiol Infect Dis 65: 247-253. doi: 10.1016/j.diagmicrobio.2009.07.022. 